\title{
Reproductive effects of 6-chloromelatonin implants and/or injections in male and female Syrian hamsters (Mesocricetus auratus)
}

\author{
M. K. Vaughan, B. A. Richardson*, L. J. Petterborg $\dagger$, G. M. Vaughan $\ddagger$ and \\ R. J. Reiter \\ Department of Cellular and Structural Biology, The University of Texas Health Science Center at \\ San Antonio, San Antonio, Texas 78284, U.S.A.
}

\begin{abstract}
Summary. Adult female hamsters were injected each afternoon for 9 weeks with $2 \cdot 5,15$ or $25 \mu \mathrm{g}$ of melatonin or 6-chloromelatonin (CM). Each drug resulted in a significant dose-related depression in uterine, ovarian and anterior pituitary gland weights. Additionally, plasma and pituitary concentrations of prolactin fell with increasing dose of either indole whereas pituitary levels of $\mathrm{LH}$ and FSH were positively correlated with dose. There was no difference in effectiveness between the two drugs.

Adult male hamsters treated for 10 weeks with daily afternoon injections of melatonin and a blank beeswax pellet had depressed testicular and accessory organ weights and plasma and pituitary concentrations of prolactin. Implantation of a $1 \mathrm{mg}$ melatonin or CM beeswax pellet in hamsters concurrently receiving daily afternoon injections of melatonin prevented the organ weight and hormonal changes, except for plasma prolactin.

Adult male hamsters treated for 10 weeks with daily afternoon injections of CM and a blank beeswax pellet had depressed reproductive organ weights and pituitary and plasma concentrations of prolactin; this depression in hormonal values and organ weights was totally prevented if the CM-injected hamsters were also bearing a beeswax-melatonin pellet.

In conclusion, 6-chloromelatonin is as effective as melatonin with regards to antigonadotrophic and counter-antigonadotrophic effects in male and female Syrian hamsters.
\end{abstract}

\section{Introduction}

The potent effects of melatonin on the neuroendocrine-reproductive axis of many mammalian species is well known (Reiter, 1980; Vaughan, 1981). Daily afternoon administration of microgram amounts of melatonin for several weeks elicits vaginal acyclicity and uterine atrophy in female Syrian hamsters (Trakulrungsi, Reiter, Trakulrungsi, Vaughan \& Waring-Ellis, 1979); similarly, the same injection protocol induces a rapid degeneration of testicular weight and function in males of this same species (Richardson et al., 1983). The antigonadotrophic consequences of daily afternoon melatonin treatment can be totally prevented if the hamsters concurrently bear a chronically releasing subcutaneous pellet containing melatonin (Reiter et al., 1977; Trakulrungsi et al., 1979); this peculiar effect of melatonin in pellet form has been called the counter-antigonadotrophic effect.

\footnotetext{
*Present address: Department of Biology, University of Michigan, Flint, MI 48503, U.S.A.

†Present address: Department of Anatomy, University of Missouri-Columbia, Columbia, MO 65212, U.S.A.

¡Present address: U.S. Army Institute of Surgical Research, Fort Sam Houston, TX 78234, U.S.A.
} 
Richardson et al. (1983) conducted a survey of natural and synthetic analogues of melatonin to determine which, if any, mimic the effects of this 5-methoxyindole in the Syrian hamster. One compound, 6-chloromelatonin, depressed gonadal weight and pituitary gonadotrophin release when administered as a single afternoon injection for 7 weeks in male hamsters. This indole is a halogenated melatonin analogue first synthesized by Flaugh, Crowell, Clemens \& Sawyer (1979). In their studies, 6-chloromelatonin blocked the ovulatory surge of luteinizing hormone (LH) and thus prevented ovulation in adult female rats. This compound has not previously been tested for antigonadotrophic activity in the female hamster. Additionally, since 6-chloromelatonin mimics the antigonadotrophic effects of melatonin in the male hamster, we wondered whether the actions of the halogenated indole extended to the counter-antigonadotrophic effects of the natural indole.

\section{Materials and Methods}

Animals and procedures. Adult male and female Syrian hamsters (Mesocricetus auratus, LAK:LVG strain, Lakeview Hamster Colony, Newfield, NJ) were housed in a windowless and environmentally controlled room $\left(22 \pm 2^{\circ} \mathrm{C}\right.$ ) illuminated (fluorescent Sylvania Super Saver 35; 1614-3328 lux) daily between $06: 00 \mathrm{~h}$ and 20:00 h. All animals were housed in clear polycarbonate cages and given food and tap water ad libitum.

Melatonin (Sigma Chemical Co., St Louis, MO) and 6-chloromelatonin (donated by J. A. Clemens, Eli Lilly Co., Indianapolis, IN) were dissolved in absolute alcohol. An aliquant of the dissolved melatonin was diluted with saline $(0.9 \%, \mathrm{w} / \mathrm{v})$ each day before injection. The indoles were injected subcutaneously daily between 16:00 h and 18:00 h. Melatonin or 6-chloromelatonin pellets were prepared by mixing $1 \mathrm{mg}$ of the respective indoles with $24 \mathrm{mg}$ softened beeswax from a local candle shop. The pellets were implanted subcutaneously on the back of ether-anaesthetized hamsters according to the treatment protocols outlined below. At the end of the experiments, the hamsters were decapitated between 08:00 and 11:00 h. Blood was collected in heparinized tubes. Plasma and pituitary samples were stored frozen at $-20^{\circ} \mathrm{C}$ until the time of assay. Weights of the gonads, accessory organs and anterior pituitary were recorded.

Experiment 1. Adult female hamsters were divided into 6 groups (9/group). They received daily subcutaneous injections of $2.5,15$ or $25 \mu \mathrm{g}$ melatonin or 6-chloromelatonin for 9 weeks. Another group of 9 animals (controls) received daily subcutaneous injections of diluent only.

Experiment 2. Adult male hamsters were divided into 4 groups (8/group) and were treated for 10 weeks. One group received daily afternoon injections of diluent and an implant of a beeswax pellet every 2 weeks. The remaining 3 groups received daily afternoon injections of melatonin $(25 \mu \mathrm{g})$ plus, every 2 weeks, a subcutaneous implant of a beeswax pellet or a pellet which contained melatonin (1 mg) or 6-chloromelatonin $(1 \mathrm{mg})$.

Expeirment 3. Adult male hamsters were divided into 4 groups (8/group) and treated for 10 weeks. Two groups received daily afternoon injections of diluent and, every 2 weeks, an implant of a beeswax pellet or a pellet containing $1 \mathrm{mg} 6$-chloromelatonin. Two other groups received daily afternoon injections of $25 \mathrm{mg}$ 6-chloromelatonin and a 2-weekly subcutaneous implant of a beeswax pellet or a pellet that contained $1 \mathrm{mg}$ melatonin.

Assays. Plasma and pituitary concentrations of LH, follicle-stimulating hormone (FSH) and prolactin were determined using antibodies, reference preparations and hormones, for iodination from the National Institute of Arthritis, Diabetes and Digestive and Kidney Disease (Bethesda, MD). LH and FSH are reported in terms of the pure hormone whereas prolactin is reported in terms of a standard pool of hamster pituitary preparation. Intra-assay variations were $9.8 \%, 6.4 \%$ and $8.8 \%$ for the prolactin, $\mathrm{LH}$ and FSH assays, respectively. Sensitivities of the assays were 
$1.2 \mathrm{ng} \mathrm{LH} / \mathrm{ml}, 3 \mathrm{ng} \mathrm{FSH} / \mathrm{ml}$ and $110 \mathrm{ng}$ prolactin (SHAP) $/ \mathrm{ml}$. All samples for a given experiment were analysed in the same assay run.

Statistical analysis. Individual group means were compared using the Bonferroni $t$ test or the Student-Newman-Keuls test to account for the multiplicity of comparisons. The relationship of hormonal response to dose and the effects of melatonin and 6-chloromelatonin were compared in Exp. 1 by using rectilinear regression of measured organ weight or hormonal value (dependent) against dose or log dose for each of the 2 drugs separately, and analysis of covariance (measured variable as dependent, log dose as covariate and drug type as grouping variable) to compare the drugs. The common slope is an index of the effectiveness of the drugs without distinguishing between them. A slope difference indicates a difference between drugs related only to certain parts of (not the overall) range of doses. A positional difference indicates a difference between drugs after accounting for effects of dose over the whole range, and is the best test for a difference in effectiveness between drugs.

\section{Results}

\section{Experiment 1}

Female hamsters treated with $2 \cdot 5,15$ or $25 \mu \mathrm{g}$ melatonin or 6-chloromelatonin had a significant log dose-related depression in uterine (Fig. 1), ovarian and anterior pituitary gland weights (Table 1); neither drug affected body weight. Plasma and pituitary concentrations of prolactin were negatively correlated with increasing dose of either indole (Fig. 1) whereas pituitary levels of LH and

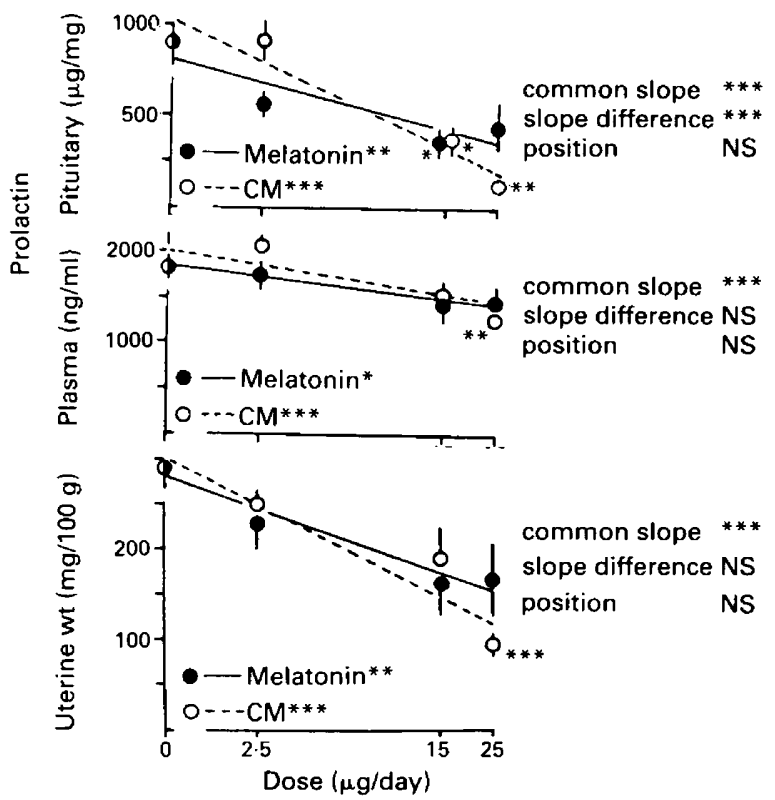

Fig. 1. Prolactin (as standard hamster anterior pituitary [SHAP] equivalent) and uterine weight (mean \pm s.e.m.) in female Syrian hamsters injected daily late in the light phase for 9 weeks with diluent (zero dose) or various doses of melatonin or 6-chloromelatonin (CM). There were 8-9 animals per dose group for each drug. Bonferroni $t$ tests compare individual group means with that of the diluent group. Single regressions for each drug (slope significance by drug symbols) including the vehicle group assigned the dose value of 1 , and covariance analyses (results on the right of each panel) excluding the vehicle group were performed using log dose values. ${ }^{*} P<0.05,{ }^{* *} P<0.01,{ }^{* *} P<0.001$. 
Table 1. Effects of various doses of melatonin or 6-chloromelatonin on ovarian and anterior pituitary measures in adult female Syrian hamsters

\begin{tabular}{|c|c|c|c|c|c|c|c|}
\hline \multirow[b]{2}{*}{ Treatment } & \multirow[b]{2}{*}{$\begin{array}{c}\text { Dose } \\
(\mu \mathrm{g})\end{array}$} & \multirow{2}{*}{$\begin{array}{c}\text { Ovarian } \\
\text { wt } \\
\text { (ng/100 } \mathrm{g} \\
\text { body wt) }\end{array}$} & \multirow{2}{*}{$\begin{array}{c}\text { Pituitary } \\
\text { wt } \\
\text { (ng/100 g } \\
\text { body wt) }\end{array}$} & \multicolumn{2}{|c|}{ LH } & \multicolumn{2}{|r|}{ FSH } \\
\hline & & & & $\begin{array}{l}\text { Plasma } \\
(\mathrm{ng} / \mathrm{ml})\end{array}$ & $\begin{array}{l}\text { Pituitary } \\
\text { (ng/mg) }\end{array}$ & $\begin{array}{l}\text { Plasma } \\
\text { (ng/ml) }\end{array}$ & $\begin{array}{l}\text { Pituitary } \\
\text { (ng/mg) }\end{array}$ \\
\hline Controls & 0 & $40.9 \pm 2.0$ & $2 \cdot 8 \pm 0 \cdot 17$ & $2 \cdot 15 \pm 0 \cdot 16$ & $386 \pm 18$ & $3.21 \pm 0.30$ & $40.7 \pm 3.7$ \\
\hline Melatonin & $\begin{array}{r}2 \cdot 5 \\
15 \cdot 0 \\
25 \cdot 0\end{array}$ & $\begin{array}{l}39 \cdot 0 \pm 1 \cdot 9 \\
35 \cdot 1 \pm 1 \cdot 0 \\
34 \cdot 6 \pm 1 \cdot 3^{b}\end{array}$ & $\begin{array}{l}2 \cdot 6 \pm 0.12 \\
2 \cdot 1 \pm 0 \cdot 13^{* *} \\
2 \cdot 1 \pm 0 \cdot 15^{* *, c}\end{array}$ & $\begin{array}{l}1.77 \pm 0 \cdot 10 \\
2 \cdot 17 \pm 0.21 \\
1.75 \pm 0.18\end{array}$ & $\begin{array}{l}289 \pm 37 \\
484 \pm 56 \\
537 \pm 84^{a}\end{array}$ & $\begin{array}{l}5.21 \pm 0.83 \\
4.38 \pm 0.34 \\
4.40 \pm 0.66\end{array}$ & $\begin{array}{l}49 \cdot 5 \pm 2 \cdot 8 \\
86 \cdot 2 \pm 8 \cdot 7^{* *} \\
84 \cdot 2 \pm 9 \cdot 2^{* *, c}\end{array}$ \\
\hline $\begin{array}{l}\text { 6-Chloro- } \\
\text { melatonin }\end{array}$ & $\begin{array}{r}2 \cdot 5 \\
15 \cdot 0 \\
25 \cdot 0\end{array}$ & $\begin{array}{l}38 \cdot 7 \pm 1 \cdot 9 \\
36 \cdot 3 \pm 1 \cdot 6 \\
35 \cdot 7 \pm 2 \cdot 0^{\mathrm{a}}\end{array}$ & $\begin{array}{l}2 \cdot 6 \pm 0.15 \\
2 \cdot 3 \pm 0 \cdot 20 \\
1 \cdot 9 \pm 0.04^{* * *, c}\end{array}$ & $\begin{array}{l}2 \cdot 0 \pm 0 \cdot 13 \\
1 \cdot 73 \pm 0 \cdot 14 \\
1 \cdot 70 \pm 0 \cdot 16^{\mathrm{a}}\end{array}$ & $\begin{array}{l}380 \pm 31 \\
517 \pm 64 \\
617 \pm 35^{* * *, c}\end{array}$ & $\begin{array}{l}4 \cdot 15 \pm 0 \cdot 61 \\
5 \cdot 16 \pm 0 \cdot 62 \\
4 \cdot 18 \pm 0 \cdot 51\end{array}$ & $\begin{array}{c}55 \cdot 3 \pm 6 \cdot 7 \\
76 \cdot 2 \pm 10 \cdot 8^{* *} \\
115.9 \pm 9.8^{* * *, c, e}\end{array}$ \\
\hline
\end{tabular}

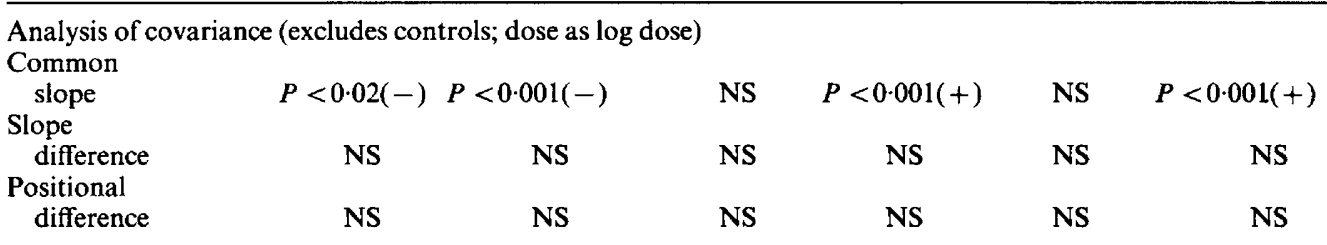

Values are means \pm s.e.m.

${ }^{*} P<0.05, P<0.01, P<0.001$ compared with control group.

${ }^{a} P<0.05 ;{ }^{b} P<0.01 ;{ }^{c} P<0.001$ : single regressions against log dose for the respective drug, including the control group arbitrarily assigned a dose value of $1(\log$ dose $=0)$.

FSH were positively correlated with dose (Table 1). Plasma concentrations of FSH were unaffected by either treatment, although the negative linear regression for plasma LH with log dose of 6-chloromelatonin was significant $(P<0 \cdot 05)$. However, analyses of covariance revealed that there were no positional differences between drugs for any of the measured variables. Lack of a significant common slope indicates further likelihood of no discernible drug effect on plasma $\mathrm{LH}$ or FSH.

\section{Experiment 2}

Adult male hamsters treated for 10 weeks with daily afternoon injections of melatonin and a blank beeswax pellet had depressed testicular $(P<0.001)$ and accessory organ weights $(P<0.001)$ (Fig. 2) compared to the saline-injected animals with blank beeswax pellets. Additionally, these animals had depressed concentrations of plasma $(P<0.001)$ and pituitary $(P<0.05)$ prolactin (Fig. 2) but normal concentrations of LH and FSH compared to the control animals. Administration of daily afternoon injections of melatonin to hamsters bearing subcutaneous pellets of melatonin or 6-chloromelatonin failed to cause regression of the male reproductive system or depression in pituitary prolactin (Fig. 2). However, these animals did have lower plasma prolactin concentrations ( $P<0.001$ in both instances), like the group receiving only the melatonin injections and blank pellets (Fig. 2). Body and anterior pituitary weights were unaffected by the treatments employed.

\section{Experiment 3}

Adult male hamsters treated for 10 weeks with daily afternoon injections of 6-chloromelatonin and a blank beeswax pellet had depressed testicular $(P<0.001)$ and pituitary $(P<0.01)$ concentrations of prolactin (Fig. 3); this depression in hormonal values was totally prevented if the 

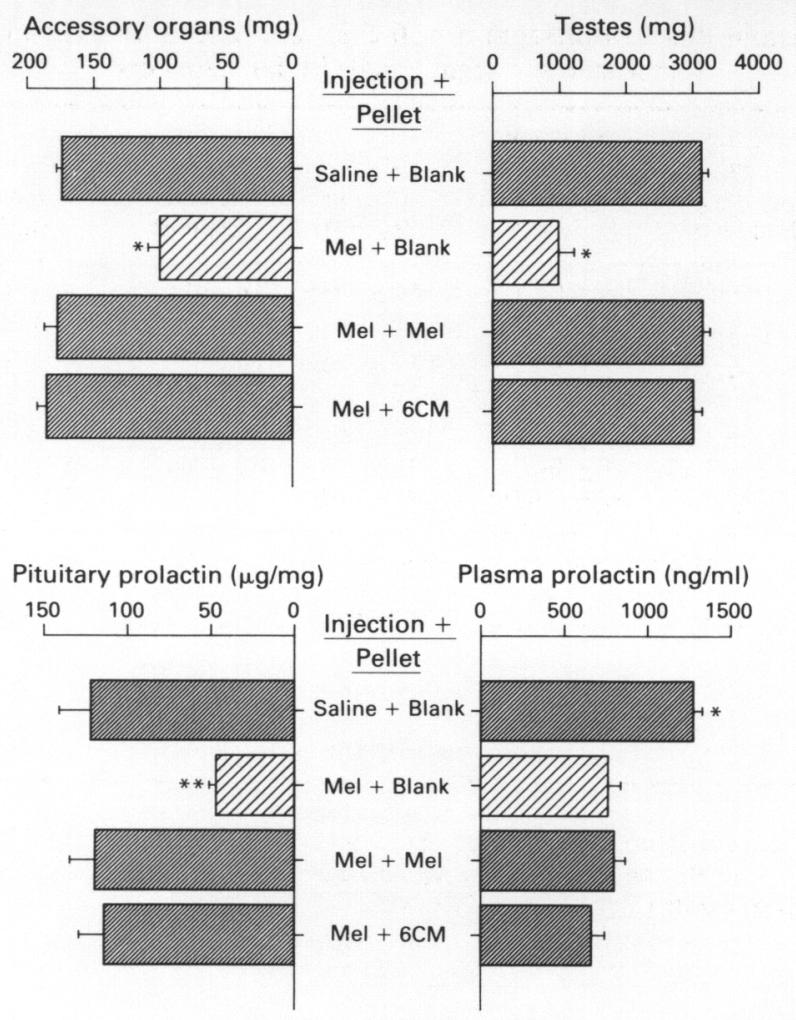

Fig. 2. Testes and accessory organ weights of and plasma and pituitary concentrations of prolactin in adult male hamsters injected for 10 weeks with $25 \mu \mathrm{g}$ melatonin (Mel) or 6-chloromelatonin (6CM) or saline diluent (saline). Additionally, every 2 weeks the animals received a subcutaneous implant of a blank $25 \mathrm{mg}$ beeswax pellet (Blank) or a beeswax pellet containing $1 \mathrm{mg}$ melatonin or 6 -chloromelatonin. Values are mean \pm s.e.m. ${ }^{*} P<0 \cdot 001$, ${ }^{* *} P<0.005$ compared with other groups.

6-chloromelatonin-injected hamsters were concurrently receiving a 2-weekly subcutaneous implant of a beeswax melatonin pellet. All hamsters treated with 6-chloromelatonin injections or pellets had significantly lower plasma concentrations of FSH. However, pituitary concentrations of FSH and LH of all animals in this experiment were within normal limits.

\section{Discussion}

Since its original description and synthesis by Flaugh et al. (1979), 6-chloromelatonin has been evaluated in only a few biological systems; these include skin lightening in the pencil-fish bioassay for melatonin (Frohn et al., 1980), inhibition of ovulation in female rats (Clemens, Flaugh, Parli \& Sawyer, 1980; Flaugh et al., 1979), reduction of testicular weight in Syrian hamsters (Richardson et al., 1983), and depression of thyroxine levels in female hamsters (Vaughan, Richardson, Petterborg, Vaughan \& Reiter, 1984). In almost every instance, the synthetic 6-chloromelatonin was equipotent to the natural indole melatonin on the endpoint measured. Such was also the case in the present studies; 6-chloromelatonin was asfective as melatonin in mimicking the antigonadotrophic and counter-antigonadotrophic effects of the natural 5-methoxyindole on gonadal and accessory organ weight and hormonal concentrations in male and female Syrian hamsters. 

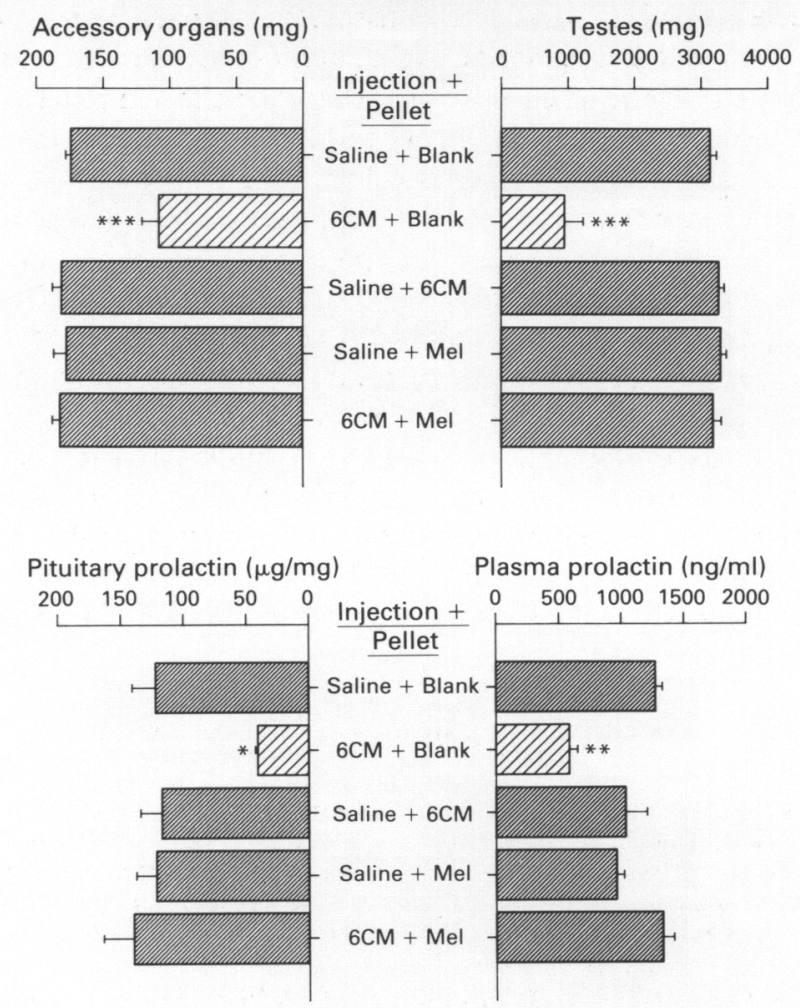

Fig. 3. Testes and accessory organ weights and plasma and pituitary concentrations of adult male hamsters injected for 10 weeks with saline diluent (saline) or $25 \mu \mathrm{g} 6$-chloromelatonin. Additionally, every 2 weeks the animals received a subcutaneous implant of $25 \mathrm{mg}$ beeswax (Blank) or a beeswax pellet containing $1 \mathrm{mg}$ melatonin (Mel). Values are mean \pm s.e.m. ${ }^{*} P<0.01,{ }^{* *} P<0.025,{ }^{* *} P<0.001$ compared with other groups.

The mechanisms by which 6-chloromelatonin evokes the same response as melatonin in the hamster are probably similar to that used by the natural indoleamine itself. The most likely site of action is at the level of the hypothalamus although other potential sites have been suggested (Vaughan, 1981). In the hypothalamus, melatonin is presumed to act on receptors which initiate a cascading series of neuronal events culminating in the hormonal and reproductive effects observed. The exact site of these receptors is unknown although one current hypothesis (Vriend, 1983) is that melatonin may inhibit TRH-releasing neurones, thereby causing a decrease in both prolactin and thyroxine. This proposal stems from several observations including: (1) TRH is a well-known stimulator of prolactin secretion, (2) plasma thyroxine concentrations are invariably depressed in hamsters treated with daily afternoon injections of melatonin, and (3) a pineal-mediated depression in the TRH content of the medial basal hypothalamus has been observed (Vriend \& Wilber, 1983).

In male and female hamsters, one of the key hormones involved in the dramatic indole-mediated involution of the gonads is prolactin. The results seen in the present experiments are typical of the depressed levels observed in melatonin-treated animals (Richardson et al., 1983; Vaughan et al., 1985). Additionally, the suppressive action of 6-chloromelatonin on pituitary prolactin concentrations corroborates previously published data from our laboratory (Richardson et al., 1983; Vaughan, Holtorf, Little, Champney \& Reiter, 1985). Previous results using other natural and synthetic analogues of melatonin have indicated that only 6-chloromelatonin and 5-methoxytryptamine are as efficacious as melatonin in the Syrian hamster (Vaughan et al., 1985). If one proposes that 
melatonin binds to a receptor localized in a target tissue, then one must presume that the receptor is only partly specific and will not distinguish all slight alterations in the structure of these indoles.

6-Chloromelatonin pellets were as effective as melatonin pellets in preventing gonadal atrophy due to afternoon melatonin injections. The theory formulated to explain this phenomenon is also contingent on the melatonin receptor hypothesis. Providing hamsters with a constant supply of melatonin (or 6-chloromelatonin) may possibly saturate the presumed receptors and keep them in a 'down-regulated' state. In such a condition, the receptors would never become sensitive to the daily pulse of exogenously administered afternoon injections; a more complete description of the down-regulation theory is presented in review articles (Reiter, 1980; Reiter, Johnson, Vaughan \& Richardson, 1981; Vaughan, 1981). The present results combining injections and pellets provide strong evidence that melatonin and 6-chloromelatonin work through the same receptor.

In summary, the results of these three experiments indicate that 6-chloromelatonin is as effective as melatonin on the organ weights and hormonal variables measured. This synthetic analogue of melatonin may prove useful in elucidating further effects of the natural indole.

\section{Supported by NSF grant No. PCM 8410592 and Project Register No. CCA 8309108.}

\section{References}

Clemens, J.A., Flaugh, M.E., Parli, J. \& Sawyer, B.D. (1980) Inhibition of luteinizing hormone release and ovulation by 6-chloro- and 6-fluoro-melatonin. Neuroendocrinology 30, 83-87.

Flaugh, M.E., Crowell, T.A., Clemens, J.A. \& Sawyer, B.D. (1979) Synthesis and evaluation of the antiovulatory activity of a variety of melatonin analogues. J. med. Chem. 22, 64-73.

Frohn, M.A., Seaborn, C.J., Johnson, D.W., Phillipou, G., Seamark, R.F. \& Matthews, C.D. (1980) Structureactivity relationship of melatonin analogues. Life Sci. 27, 2043-2046.

Reiter, R.J. (1980) The pineal and its hormones in the control of reproduction. Endocr. Rev. 1, 109-131.

Reiter, R.J., Johnson, L.Y., Vaughan, M.K. \& Richardson, B.A. (1981) Pineal constituents and reproductive physiology. Prog. clin. biol. Res. 74, 163-178.

Reiter, R.J., Rudeen, P.K., Sackman, J.W., Vaughan, M.K., Johnson, L.Y. \& Little, J.C. (1977) Subcutaneous melatonin implants inhibit reproductive atrophy in male hamsters induced by daily melatonin injections. Endocr. Res. Commun. 4, 35 44.

Richardson, B.A., Vaughan, M.K., Petterborg, L.J., Johnson, L.Y., King, T.S., Smith, I. \& Reiter, R.J. (1983) Natural and synthetic analogues of melatonin and related compounds. I. Effects on the reproductive system of the male Syrian hamster. $J$. Neural Transm. 56, 187-197.
Trakulrungsi, C., Reiter, R.J., Trakulrungsi, W.K., Vaughan, M.K. \& Waring-Ellis, P.J. (1979) Interaction of daily injections and subcutaneous reservoirs of melatonin on the reproductive physiology of female Syrian hamsters. Acta endocr., Copenh. 91, 59-69.

Vaughan, M.K. (1981) The pineal gland-a survey of its antigonadotrophic substances and their actions. Int. Rev. Physiol. 24, 41-95.

Vaughan, M.K., Richardson, B.A., Petterborg, L.J., Vaughan, G.M. \& Reiter, R.J. (1984) Injections and/ or implants of 6-chloromelatonin and melatonin: Effects on plasma thyroid hormones in male and female Syrian hamsters. Biomed. Res. 5, 413-418.

Vaughan, M.K., Holtorf, A.P., Little, J.C., Champney, T.H. \& Reiter, R.J. (1985) A survey of pineal indoles and analogues which effect prolactin secretion in the adult male Syrian hamster. In Prolactin: Basic and Clinical Correlates, pp. 143-150. Eds R. M. MacLeod, M. O. Thorner \& U. Scapagnini. Liviana, Padova.

Vriend, J. (1983) Pineal-thyroid interactions. Pineal Res. Rev. 1, 183-206.

Vriend, J. \& Wilber, J.F. (1983) Influence of the pineal gland on hypothalamic content of TRH in the Syrian hamster. Horm. Res. 17, 108-113.

Received 8 January 1986 